\title{
Metallicity of M dwarfs
}

\section{A photometric calibration and the impact on the mass-luminosity relation at the bottom of the main sequence ${ }^{\star, \star \star}$}

\author{
X. Bonfils ${ }^{1,2}$, X. Delfosse ${ }^{1}$, S. Udry ${ }^{2}$, N. C. Santos ${ }^{2,3}$, T. Forveille ${ }^{1,4}$, and D. Ségransan ${ }^{2}$ \\ 1 Laboratoire d'Astrophysique, Observatoire de Grenoble, BP 53, 38041 Grenoble Cedex 9, France \\ e-mail: Xavier.Bonfils@obs.ujf-grenoble.fr \\ 2 Observatoire de Genève, $51 \mathrm{ch}$. des Maillettes, 1290 Sauverny, Switzerland \\ 3 Centro de Astronomia e Astrofisica da Universidade de Lisboa, Observatório Astrónomico de Lisboa, Tapada de Ajuda, 1349-018 Lisboa, \\ Portugal \\ ${ }^{4}$ Canada-France-Hawaii Telescope Corporation, 65-1238 Mamalahoa Highway, Kamuela, HI 96743, Hawaii, USA
}

Received 11 March 2005 / Accepted 19 May 2005

\section{ABSTRACT}

We obtained high resolution ELODIE and CORALIE spectra for both components of 20 wide visual binaries composed of an F-, G- or Kdwarf primary and an M-dwarf secondary. We analyse the well-understood spectra of the primaries to determine metallicities ([Fe/H]) for these 20 systems, and hence for their $\mathrm{M}$ dwarf components. We pool these metallicities with determinations from the literature to obtain a precise $( \pm 0.2$ dex $)$ photometric calibration of $\mathrm{M}$ dwarf metallicities. This calibration represents a breakthrough in a field where discussions have had to remain largely qualitative, and it helps us demonstrate that metallicity explains most of the large dispersion in the empirical $V$-band massluminosity relation. We examine the metallicity of the two known M-dwarf planet-host stars, Gl 876 (+0.02 dex) and Gl 436 (-0.03 dex), in the context of preferential planet formation around metal-rich stars. We finally determine the metallicity of the 47 brightest single $\mathrm{M}$ dwarfs in a volume-limited sample, and compare the metallicity distributions of solar-type and M-dwarf stars in the solar neighbourhood.

Key words. techniques: spectroscopic - stars: abundances - stars: late-type - binaries: visual - planetary systems stars: individual: Gl 876, Gl 436

\section{Introduction}

The very low mass $\mathrm{M}$ dwarfs are small, cool and faint, but they dominate the Galaxy by number $(\sim 50-70 \%)$, and even by total mass $(\sim 70 \%)$ (Chabrier 2003). Any realistic model of the Galaxy therefore needs an excellent description of this faint component. Over the last decade, stellar models of very low mass stars have made great strides, but they still have to use some incomplete or approximate input physics (Chabrier $\&$ Baraffe 2000). Descriptions of these stars therefore need a strong empirical basis, or validation.

In Ségransan et al. (2003) and Delfosse et al. (2000, hereafter DFS00), we have validated the model predictions for radii and luminosities. The empirical radii match the models very well, and have no dispersion beyond the measurement errors. The infrared mass-luminosity (hereafter $\mathrm{M} / \mathrm{L}$ ) relations also have negligible dispersion, and similarly agree with model

$\star$ Based on observations obtained with the ELODIE spectrograph on the $1.93 \mathrm{~m}$ telescope of the Observatoire de Haute Provence, France.

$\star \star$ Tables 4 and 5 are only available in electronic form at http://www.edpsciences.org predictions. The $V$-band $\mathrm{M} / \mathrm{L}$ relation, in contrast, has a large $(\sim \pm 1 \mathrm{mag})$ intrinsic scatter. In DFS00 we suggested that metallicity might explain most of this intrinsic dispersion, but for lack of quantitative metallicity estimates we could not pursue this suggestion.

M-dwarf metallicities have also become relevant in the context of planet formation around very low mass stars. One robust result of the exoplanet searches is that $G$ and $K$ stars which host planets are on average more metal-rich than the bulk of the solar neighbourhood population (Gonzalez 1997; Santos et al. 2001, 2003, 2004). A leading explanation for this observation is that the disks of metal-rich stars contain larger amounts of refractory dust, and that more massive dust disks are much more likely to form planets. This has a clear bearing on planets around M dwarfs, since these low mass stars are likely to have smaller disks than solar-type stars of the same metallicity. Assuming that protostellar disk mass scales with stellar mass and within the core-accretion scenario, Laughlin et al. (2004) and Ida \& Lin (2005) show that formation of Jupiter-mass planets is seriously inhibited around the less massive $\mathrm{M}$ dwarfs $\left(M_{\star}<0.4 M_{\odot}\right)$. To date, the only two M dwarfs known to host 
planets are Gl 876 (Delfosse et al. 1998; Marcy et al. 1998, 2001) and Gl 436 (Butler et al. 2004), but a number of ongoing surveys are looking for more (e.g. Bonfils et al. 2004; Endl et al. 2003; Kuerster et al. 2003; Wright et al. 2004). They will bring new constraints on the frequency of planets as a function of stellar mass, and metallicity will be one of the key parameters in the comparison with solar-type targets.

Measuring M-dwarf metallicities from their spectra is unfortunately difficult. As the spectral subtype increases, the atmospheres of these cool stars $\left(\sim 3800 \mathrm{~K}(\mathrm{M} 0)>T_{\text {eff }}>\sim 2100 \mathrm{~K}\right.$ (M9)) contain increasingly abundant diatomic and triatomic molecules ( $\mathrm{TiO}, \mathrm{VO}, \mathrm{H}_{2} \mathrm{O}, \mathrm{CO}, \mathrm{FeH}, \mathrm{CrH} .$. ), which spectroscopically defines the $\mathrm{M}$ class. These components have complex and extensive absorption band structures, which eventually leave no continuum point in the spectrum. In a late-M dwarf, the local pseudo-continuum estimated from a high resolution spectrum is defined by a forest a weak lines, and often underestimates the true continuum by a factor of a few. The "line-by-line" spectroscopic analysis used for hotter stars therefore becomes impossible for late-M dwarfs, and a full spectral synthesis must be used. Besides the practical complexities of that approach, the atmospheric models do not yet reproduce the details of high resolution spectra (mostly due to limitations of their molecular opacity databases). This therefore leaves some doubt about the reliability of the resulting metallicities. Here we instead observe visual binaries that contain both an M-dwarf and a solar-type star. They presumably share a common metallicity that reflects the composition of their parent molecular cloud, and we use the much better understood spectrum of the solar-type star to infer the metallicity of the M dwarf.

In Sect. 2 we review the limited literature on observational M-dwarf metallicities. Section 3 briefly describes the binary sample, the observations, and our analysis of the primary star spectra. Section 4 describes the derivation of a photometric metallicity estimator for very low-mass stars. Section 5 reexamines the dispersion of the $V$-band $\mathrm{M} / \mathrm{L}$ relation in the light of the new metallicities and proposes a more precise mass-metallicity-luminosity relation for very low-mass stars. In Sect. 6 we apply the metallicity estimator to the two known Mdwarf planet-host stars. Section 7 lists estimated metallicities for a volume-limited sample of northern $\mathrm{M}$ dwarfs, and compares its metallicity distribution with that of nearby solar-type stars.

\section{Previous metallicity estimates of M dwarfs}

The first attempt to measure metallicities for $M$ dwarfs was by Mould (1976, 1978) who performed line-by-line analyses of atomic lines in intermediate resolution near-IR spectra of a few stars, using model atmospheres available then. Jones et al. (1996) used a similar approach, but with the benefit of atmospheric structures from an early version of the modern PHOENIX code (Allard \& Hauschildt 1995). Gizis (1997) matched low resolution optical spectra to synthetic spectra from the same Allard \& Hauschildt (1995) models, and derived relatively crude metallicities that allowed them to classify $\mathrm{M}$ dwarfs into 3 broad categories (dwarfs, subdwarfs and extreme subdwarfs). Gizis \& Reid (1997) validated that metallicity scale with observations of binary stars containing one M-dwarf component and one warmer star.

Valenti et al. (1998) performed detailed spectral synthesis of a very high resolution spectrum of G1725 B (vB 10) to determine its atmospheric parameters. Zboril \& Byrne (1998) matched high resolution red spectra (5500-9000 $)$ of $7 \mathrm{~K}$ and $11 \mathrm{M}$ dwarfs to Allard \& Hauschildt (1995) synthetic spectra. They conclude that for the $\mathrm{M}$ dwarfs the resulting metallicities are only indicative, a conclusion that probably applies to most previous references, at least for the later subtypes. Jones et al. (2002) synthesized the water vapor bands for Infrared Space Observatory (ISO) $2.5-3 \mu \mathrm{m}$ spectra of $3 \mathrm{M}$ dwarfs to derive their parameters.

The limited overlap between these studies shows that they have not yet converged to consistency. G1 725 B was measured by both Valenti et al. (1998) and Zboril \& Byrne (1998), who respectively derive $[\mathrm{M} / \mathrm{H}]=-0.92$ and $[\mathrm{M} / \mathrm{H}]=-0.15$. Jones et al. (1996, 2002), Zboril \& Byrne (1998) and Dawson \& De Robertis (2004) all measured Barnard's star and found subsolar to solar metallicity, but the values spread from -0.75 to 0.0 dex. Kapteyn's star is also consistently found to be submetallic (Mould 1976; Jones 2002; Woolf \& Wallerstein 2004, 2005), as expected from its population II kinematics but again with some dispersion.

The above references attempt to simultaneously determine the effective temperature $\left(T_{\text {eff }}\right)$, the gravity $(\log g)$ and the metallicity $([\mathrm{M} / \mathrm{H}])$, by minimizing the difference between observed and model spectra. Unfortunately the 3 parameters are strongly coupled, in particular for spectra that are not flux calibrated. Furthermore, the models do not yet reproduce the observed spectra in perfect detail, mostly due to remaining shortcomings in their molecular transition databases, especially for the later subtypes. The interpretation therefore involves estimations as to which features of the spectra should be ignored and which should be given maximum weight, and to some extent the process remains an art. Different practitioners would likely obtain somewhat different answers from the same data and atmospheric models, and they definitely do when they analyse different spectral bands observed with different spectral resolutions.

The recent analyses of Kapteyn's and Barnard's stars by Woolf \& Wallerstein (2004) and Dawson \& De Robertis (2004), by contrast, are anchored in model-independent $T_{\text {eff }}$ and $\log g$ values from Ségransan et al. (2003). Ségransan et al. (2003) combined their interferometric radius measurements with the bolometric flux to determine $T_{\text {eff }}$, reversing the more usual procedure of determining stellar radii from effective temperature and luminosity, and they computed the gravity from the linear radius and a mass derived from the well constrained $K$ band mass-luminosity relation. Woolf \& Wallerstein (2004) and Dawson \& De Robertis (2004) could therefore concentrate the full information content of their spectra on determining the metallicity, free of any uncontrolled coupling with the other atmospheric parameters.

Woolf \& Wallerstein (2005, hereafter WW05) analysed a much larger sample of $35 \mathrm{~K}$ and $\mathrm{M}$ dwarfs, that for now do not have interferometric radius measurements. 
Table 1. Observed visual binaries with an M-dwarf secondary.

\begin{tabular}{|c|c|c|c|c|c|c|c|c|c|}
\hline \multicolumn{5}{|c|}{ Primaries } & \multicolumn{5}{|c|}{ Secondaries } \\
\hline Name & $\alpha(2000)$ & $\delta(2000)$ & $m_{V}$ & Sp. Typ. & Name & $\alpha(2000)$ & $\delta(2000)$ & $m_{V}$ & Sp. Typ. \\
\hline GJ 1021 & $00: 45: 45.593$ & $-47: 33: 07.15$ & 5.80 & G1V & CD-48 176 B & $00: 45: 43.5$ & $-47: 33: 00$ & 13.5 & $\mathrm{M}$ \\
\hline Gl34 A & 00:49:06.291 & $+57: 48: 54.67$ & 3.44 & F9V & Gl 34 B & $00: 49: 06.5$ & $+57: 48: 55$ & 7.51 & K7 \\
\hline Gl 53.1 A & 01:07:37.872 & $+22: 57: 17.91$ & 8.41 & $\mathrm{~K} 4 \mathrm{~V}$ & G153.1 B & 01:07:37.7 & $+22: 57: 18$ & 13.60 & M3 \\
\hline Gl 81.1A & 01:57:09.607 & $-10: 14: 32.75$ & 6.42 & G5 & Gl 81.1B் & 01:57:11.1 & $-10: 14: 53$ & 11.21 & K7 \\
\hline Gl $105 \mathrm{~A}$ & 02:36:04.894 & $+06: 53: 12.73$ & 5.79 & $\mathrm{~K} 3 \mathrm{~V}$ & G1 $105 \mathrm{~B}$ & $02: 36: 15.3$ & $+06: 52: 19$ & 11.68 & M4 \\
\hline G1 107 A & 02:44:11.986 & $+49: 13: 42.41$ & 4.10 & F7V & Gl 107 B & $02: 44: 11.8$ & $+49: 13: 43$ & 10.06 & M1.5 \\
\hline GJ 3194 A & 03:04:09.636 & $+61: 42: 20.99$ & 6.64 & G4V & GJ 3195 B & 03:04:43.6 & $+61: 44: 08$ & 12.5 & M3 \\
\hline Gl 166 A & $04: 15: 16.320$ & $-07: 39: 10.33$ & 4.43 & $\mathrm{~K} 1 \mathrm{~V}$ & G1 $166 \mathrm{C}$ & $04: 15: 18.5$ & $-07: 39: 07$ & 11.17 & M4.5 \\
\hline G1 211 & $05: 41: 20.336$ & $+53: 28: 51.81$ & 6.21 & $\mathrm{~K} 1$ & Gl 212 & $05: 41: 30.7$ & $+53: 29: 23$ & 9.80 & M0.5 \\
\hline Gl 231.1 A & $06: 17: 16.138$ & $+05: 06: 00.40$ & 5.70 & F9V & Gl 231.1 B & $06: 17: 11.0$ & $+05: 07: 06$ & 13.42 & M3.5 \\
\hline G1 $250 \mathrm{~A}$ & $06: 52: 18.050$ & $-05: 10: 25.37$ & 6.59 & $\mathrm{~K} 3$ & G1 250 B & $06: 52: 18.1$ & $-05: 11: 26$ & 10.09 & M2 \\
\hline G1 297.2 A & $08: 10: 39.826$ & $-13: 47: 57.15$ & 5.53 & F7 & Gl 297.2 B & $08: 10: 34.0$ & $-13: 48: 48$ & 11.80 & M2 \\
\hline Gl 324 A & $08: 52: 35.811$ & $+28: 19: 50.95$ & 5.53 & G8 & Gl 324 B & $08: 52: 40.8$ & $+28: 18: 59$ & 13.14 & M3.5 \\
\hline G1 $505 \mathrm{~A}$ & $13: 16: 51.052$ & $+17: 01: 01.86$ & 6.69 & $\mathrm{~K} 2$ & G1 505 B & $13: 16: 51.7$ & $+17: 00: 56$ & 9.6 & M0.5 \\
\hline Gl 544 A & $14: 19: 34.864$ & $-05: 09: 04.30$ & 7.58 & $\mathrm{~K} 1$ & G1544 B & $14: 19: 35.0$ & $-05: 09: 08$ & 14.1 & M6 \\
\hline NLTT 45789 & 18:00:38.894 & $+29: 34: 18.91$ & 7.07 & G2V & NLTT 45791 & 18:00:45.4 & $+29: 33: 57$ & 13.1 & M \\
\hline G1 768.1 A & 19:51:01.643 & $+10: 24: 56.62$ & 5.12 & F8V & G1768.1 B & 19:51:01.1 & $+10: 24: 43$ & 13.1 & M3.5 \\
\hline Gl 783.2 A & 20:11:06.074 & $+16: 11: 16.80$ & 7.34 & K1V & Gl 783.2 B & $20: 11: 13.4$ & $+16: 11: 07$ & 13.94 & M4 \\
\hline G1797 A & $20: 40: 45.141$ & $+19: 56: 07.93$ & 6.43 & G5V & G1797 B & $20: 40: 44.4$ & $+19: 53: 59$ & 11.88 & $\mathrm{M} 2.5$ \\
\hline Gl 806.1 A & $20: 46: 12.683$ & $+33: 58: 12.92$ & 2.48 & K0III & Gl 806.1 B & $20: 46: 12.7$ & $+33: 58: 12$ & 13.4 & M3 \\
\hline G1 $872 \mathrm{~A}$ & $22: 46: 41.581$ & $+12: 10: 22.40$ & 4.20 & F7V & G1 872 B & $22: 46: 41.6$ & $+12: 10: 20$ & 11.7 & M1 \\
\hline
\end{tabular}

WW05 therefore rely on photometric effective temperatures ( $\left.T_{\text {eff }}\right)$ and they use a photometric radius to compute the gravity. While less direct than the Ségransan et al. (2003) measurements, this procedure rests on relations which that paper validates, and that in our view is currently preferable to determining those parameters from the spectrum. The 15 Woolf \& Wallerstein (2005) M dwarfs are overwhelmingly of early subtypes (only one is later than M 1.5) and they concentrate on low metallicity targets. Their spectra therefore have limited molecular veiling. Together with their use of the latest generation of the PHOENIX models, this reduces their sensitivity to the remaining shortcomings of the molecular opacity databases. The parameter space which they cover complements our own measurements, and we make extensive use of these data in our discussion.

\section{Observations and analysis}

\subsection{Sample, observation and data reduction}

We selected wide physical visual binaries composed of an F-, G- or K-primary component and an M-dwarf secondary, from the Gliese \& Jareiss (1991) catalogue of nearby stars, the Poveda et al. (1994) catalogue of wide-binary and multiple systems of nearby stars, and the Gould \& Chanamé (2004) list of physical HIPPARCOS binaries. We further required that the components be separated by at least $5^{\prime \prime}$ and that the secondary be brighter than $V=14$, to facilitate observations of the faint $\mathrm{M}$ dwarf. Fast rotators, double-lined spectroscopic binaries (SB2) and close visual binaries were rejected a priori when known, and otherwise discarded a posteriori. These criteria resulted in a list of 70 pairs.
Table 2. New double-lined spectroscopic binaries.

\begin{tabular}{llll}
\hline \hline Name & HD & $\alpha(2000)$ & $\delta(2000)$ \\
\hline GJ 3409 B & 263175 B & $06: 46: 07.6$ & $+32: 33: 13.2$ \\
G1 771 B & - & $19: 55: 18.8$ & $+06: 24: 36$ \\
\hline
\end{tabular}

We discarded 2 systems whose secondaries (GJ 3409 B and G1 771 B) were SB2 binaries (reported in Table 2). The Gl 549 system had to be rejected as the F7V primary is a fast rotator $\left(v \sin i \sim 50 \mathrm{~km} \mathrm{~s}^{-1}\right)$. The Gl 695 system was also rejected as both components are themselves close visual binaries. Here we analyse 21 of those systems (Table 1), of which 20 have M-dwarf secondaries (the last one being classified as K7V).

Most of the spectra were gathered using the ELODIE spectrograph (Baranne et al. 1996) on the 1.93-m telescope of Observatoire de Haute-Provence (France). ELODIE covers a visible spectral range from 3850 to $6800 \AA$ with a resolution of 45000 . For GJ 1021, Gl 166 A and Gl 250 A we reuse spectra observed by Santos et al. (2001) with the CORALIE spectrograph (1.20-m Swiss Telescope, La Silla Observatory ESO, Chile). CORALIE has a slightly wider spectral range than ELODIE, 3650 to $6900 \AA$, and a slightly higher resolution of 50000 . On-line processing is integrated with control software of both spectrographs, and automatically produces optimally extracted, flat-fielded and wavelength calibrated spectra, with algorithms described in Baranne et al. (1996). For all primaries the present observations used the "Object-only" mode of the spectrograph, where its optional reference fiber is not illuminated. This mode provides optimal scattered light correction, at the cost of degraded radial velocity precision $\left(\sim 100 \mathrm{~m} \mathrm{~s}^{-1}\right)$. The wavelength calibration used a single Thorium-Argon exposure obtained at the beginning of each night. For each primary we 
Table 3. Stellar parameters measured on the primaries. $[\mathrm{Fe} / \mathrm{H}]$ applies for both components.

\begin{tabular}{|c|c|c|c|c|c|c|}
\hline \multicolumn{2}{|c|}{ Primary } & \multirow[t]{2}{*}{ Secondary } & \multicolumn{4}{|c|}{ Stellar parameters measured on the primaries } \\
\hline Gliese/NLTT & $\mathrm{HD}$ & & $T_{\text {eff }}$ & $\log (g)$ & $v_{t}$ & {$[\mathrm{Fe} / \mathrm{H}]$} \\
\hline GJ 1021 & 4391 & CD-48 176B & $5967 \pm 70$ & $4.74 \pm 0.14$ & $1.39 \pm 0.17$ & $\overline{-\mathbf{0 . 0 8} \pm 0.09}$ \\
\hline G134 A & 4614 & G134 B & $5895 \pm 68$ & $4.43 \pm 0.13$ & $1.37 \pm 0.22$ & $-\mathbf{0 . 3 1} \pm 0.09$ \\
\hline Gl 53.1A & 6660 & GI 53.1 B & $4705 \pm 131$ & $4.33 \pm 0.26$ & $0.76 \pm 0.25$ & $+\mathbf{0 . 0 7} \pm 0.12$ \\
\hline Gl 81.1 A & 11964 & GI 81.1 B & $5311 \pm 42$ & $3.97 \pm 0.08$ & $0.86 \pm 0.05$ & $+\mathbf{0 . 0 9} \pm 0.06$ \\
\hline Gl $105 \mathrm{~A}$ & 16160 & Gl 105 B & $4846 \pm 65$ & $4.29 \pm 0.13$ & $0.81 \pm 0.10$ & $\mathbf{- 0 . 1 9} \pm 0.07$ \\
\hline Gl 107 A & 16895 & Gl 107 B & $6328 \pm 86$ & $4.43 \pm 0.17$ & $1.73 \pm 0.31$ & $-\mathbf{0 . 0 3} \pm 0.09$ \\
\hline GJ 3194 A & 18757 & GJ 3195 B & $5681 \pm 34$ & $4.49 \pm 0.06$ & $1.01 \pm 0.07$ & $-\mathbf{0 . 3 1} \pm 0.04$ \\
\hline Gl 166 A & 26965 & Gl 166 C & $5125 \pm 56$ & $4.43 \pm 0.11$ & $0.30 \pm 0.15$ & $-\mathbf{0 . 3 3} \pm 0.06$ \\
\hline Gl 211 & 37394 & GI 212 & $5293 \pm 109$ & $4.50 \pm 0.21$ & $0.79 \pm 0.17$ & $+\mathbf{0 . 0 4} \pm 0.11$ \\
\hline G1 231.1 A & 43587 & GI 231.1 B & $5946 \pm 32$ & $4.38 \pm 0.06$ & $1.15 \pm 0.06$ & $\mathbf{- 0 . 0 2} \pm 0.04$ \\
\hline Gl $250 \mathrm{~A}$ & 50281 & GI 250 B & $4670 \pm 80$ & $4.41 \pm 0.16$ & $0.70 \pm 0.19$ & $\mathbf{- 0 . 1 5} \pm 0.09$ \\
\hline Gl 297.2 A & 68146 & GI 297.2 B & $6280 \pm 106$ & $4.46 \pm 0.21$ & $1.81 \pm 0.26$ & $-\mathbf{0 . 0 9} \pm 0.09$ \\
\hline G1 $324 \mathrm{~A}$ & 75732 & GI 324 B & $5283 \pm 59$ & $4.36 \pm 0.11$ & $0.87 \pm 0.08$ & $+\mathbf{0 . 3 2} \pm 0.07$ \\
\hline G1505 A & 115404 & Gl 505 B & $4983 \pm 48$ & $4.41 \pm 0.09$ & $0.84 \pm 0.07$ & $\mathbf{- 0 . 2 5} \pm 0.05$ \\
\hline G1544 A & 125455 & Gl 544 B & $5271 \pm 189$ & $4.85 \pm 0.37$ & $0.87 \pm 0.36$ & $-\mathbf{0 . 2 0} \pm 0.19$ \\
\hline NLTT 45789 & 164595 & NLTT 45791 & $5696 \pm 41$ & $4.36 \pm 0.08$ & $0.83 \pm 0.06$ & $\mathbf{- 0 . 0 7} \pm 0.05$ \\
\hline Gl 768.1 A & 187691 & GI 768.1 B & $6248 \pm 93$ & $4.63 \pm 0.18$ & $2.36 \pm 0.48$ & $+\mathbf{0 . 0 7} \pm 0.12$ \\
\hline G1 783.2 A & 191785 & GI 783.2 B & $5094 \pm 66$ & $4.31 \pm 0.13$ & $0.30 \pm 0.19$ & $\mathbf{- 0 . 1 6} \pm 0.08$ \\
\hline Gl 797 A & $197076 \mathrm{~A}$ & Gl 797 B & $5889 \pm 32$ & $4.59 \pm 0.06$ & $1.01 \pm 0.06$ & $-\mathbf{0 . 0 7} \pm 0.04$ \\
\hline G1 806.1 A & 197989 & Gl 806.1 B & $4911 \pm 85$ & $2.98 \pm 0.17$ & $1.61 \pm 0.08$ & $\mathbf{- 0 . 0 5} \pm 0.13$ \\
\hline Gl $872 \mathrm{~A}$ & 215648 & GI 872 B & $6156 \pm 99$ & $4.09 \pm 0.19$ & $4.05 \pm 2.01$ & $-\mathbf{0 . 3 6} \pm 0.11$ \\
\hline
\end{tabular}

recorded a sequence of 3 spectra, and applied a median filter to remove any unflagged cosmic ray hit. The combined spectra have signal-to-noise ratios of approximately 200 per pixel ( $\sim 300$ per resolution element), amply sufficient for our spectroscopic analysis. We also obtained spectra for the secondaries, usually with a much lower signal to noise ratio, from which we planned to derive spectroscopic metallicity diagnostics that can be applied at moderate/low signal to noise ratio data. That goal has proved more difficult than we expected, and it will be discussed in a future paper if we are successful.

\subsection{Spectroscopic analysis}

Our spectroscopic analysis of the primaries follows the procedure described by Santos et al. (2004) for planet host stars. Briefly, we used the Gaussian fitting procedure of the IRAF splot task to the measure equivalent widths $\left(W_{\lambda}\right)$ of $39 \mathrm{Fe} \mathrm{I}$ and 12 Fe II lines. The stellar parameters were then derived using the 2002 version of the MOOG code (Sneden 1973), and a grid of Kurucz (1993) ATLAS9 model atmospheres. Table 3 presents the resulting atmospheric parameters (microturbulence, effective temperature $\left(T_{\text {eff }}\right)$, surface gravity and iron abundance $([\mathrm{Fe} / \mathrm{H}])$, which we use as a measurement of the overall metallicity $([\mathrm{M} / \mathrm{H}])$.

The standard errors on $T_{\text {eff }}, \log g, \xi_{t}$ and $[\mathrm{Fe} / \mathrm{H}]$ were derived as described in Santos et al. (2004), following the prescriptions of Gonzalez \& Vanture (1998). The resulting uncertainties are internal, in the sense that they ignore possible scale offsets. There is currently some disagreement on e.g. the apropriate temperature scale for solar-type dwarfs, as well as which model atmospheres better reproduce the real stellar atmospheres. The true errors may consequently be larger, but the listed standard errors are appropriate for comparisons within our sample. As discussed in Santos et al. (2004, 2005), the method and the grid of ATLAS9 atmospheres used gives excellent results, compatible with those derived by other authors using other model atmospheres and methods to derive the stellar parameters and metallicities.

Six of the observed primaries have published stellar parameters (Santos et al. 2004; Edvardsson et al. 1993). Comparison of our determinations of $T_{\text {eff }}$ and $[\mathrm{Fe} / \mathrm{H}]$ with these litterature values (Fig. 1) shows that they agree to within the stated errors.

\section{A photometric calibration of M-dwarf metallicities}

From this point on, we use a sample (Table 4) that combines our own metallicity measurements with those of WW05. As discussed above, we expect the latter to be reliable, and they reach to lower metallicities for mostly hotter atmospheres. The two datasets are therefore complementary, but they have enough overlap in the $\left(T_{\text {eff }},[\mathrm{Fe} / \mathrm{H}]\right)$ plane to assess possible systematic differences (Fig. 2). Table 4 contains all WW05 stars with known parallaxes, except LHS 1138, listed as a G5 dwarf in SIMBAD, and GJ 1064 D, a clear outlier in our relations and perhaps a photometric binary. We restrict the analysis to stars brighter than $M_{K}=7.5$, since the sampling is very sparse for fainter stars.

The left panel of Fig. 2 displays the effect of metallicity in the $M_{K}$ vs. $V-K$ observational Hertzsprung-Russell diagram, with symbol sizes proportional to the metallicity of the corresponding stars. After experimenting with several colourmagnitude diagrams, we found that amongst commonly available photometric bands this combination maximizes the metallicity sentivity. It is immediately obvious that lower metallicity stars are much bluer at a given absolute $M_{K}$ magnitude, and we 

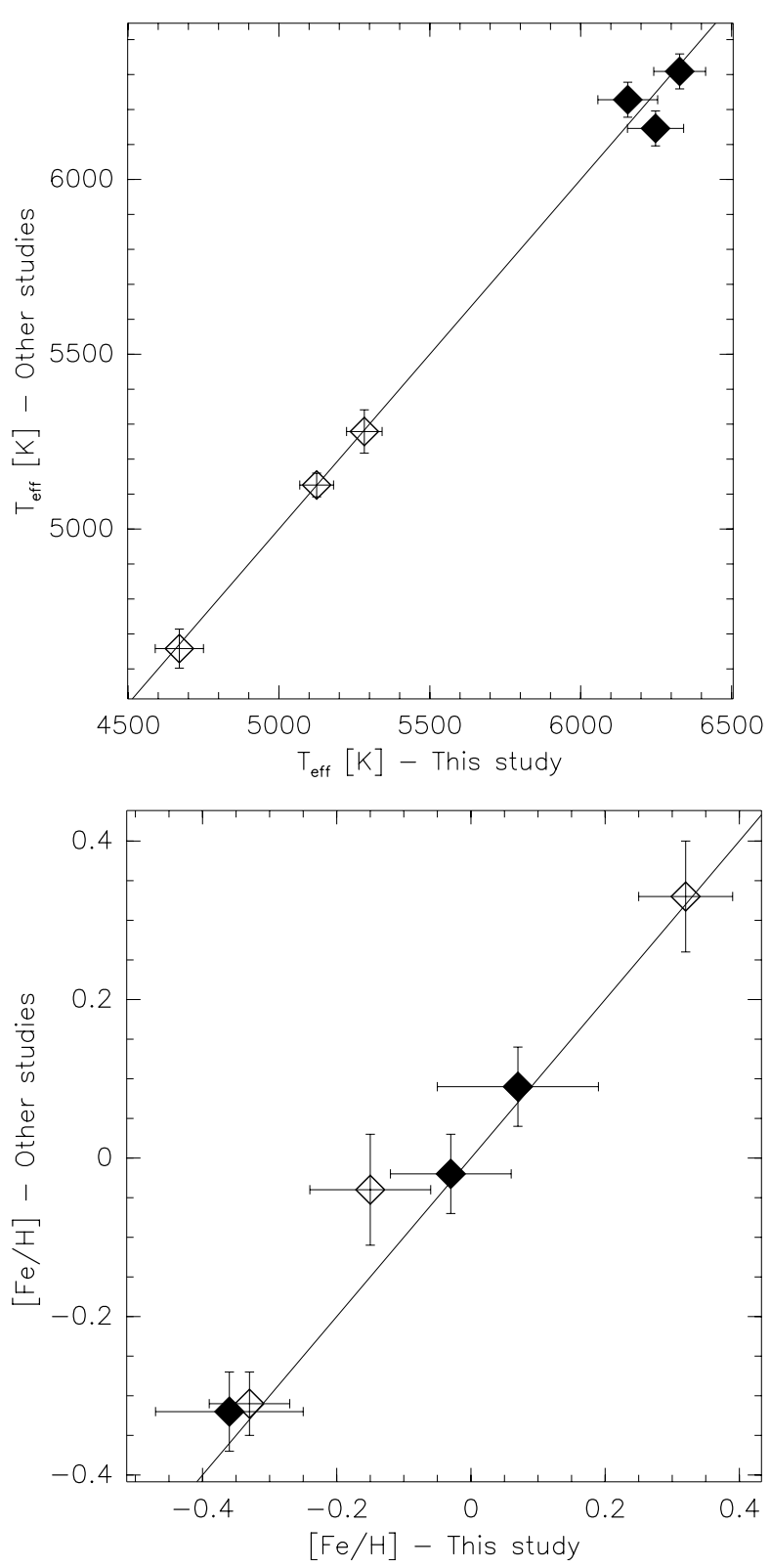

Fig. 1. Comparison of $T_{\text {eff }}$ (upper panel) and [Fe/H] (lower panel) between our study and Santos et al. (2004, open diamonds) and Edvardsson et al. (1993, filled diamonds). The errorbars represent the individual $1 \sigma$ errors for our study and for Santos et al. Edvardsson et al. do not list individual error estimates and we adopt their typical errors of $50 \mathrm{~K}$ for $T_{\text {eff }}$ and $0.05 \mathrm{dex}$ for $[\mathrm{Fe} / \mathrm{H}]$. The over-plotted lines represent an identity relation, not a fit to the data.

find the metallicity well described by the following polynomial relation between $M_{K}$ and $V-K$ :

$$
\begin{aligned}
{[\mathrm{Fe} / \mathrm{H}]=} & 0.196-1.527 M_{K}+0.091 M_{K}^{2} \\
& +1.886(V-K)-0.142(V-K)^{2},
\end{aligned}
$$

valid for $M_{K} \in[4,7.5], V-K \in[2.5,6]$ and $[\mathrm{Fe} / \mathrm{H}] \in$ $[-1.5,+0.2]$ and with an observed dispersion of only 0.2 dex.

Part of this dispersion might be due to a few of the higher mass stars having evolved slightly off the main sequence. For instance between $8 \mathrm{Gyr}$ and $10 \mathrm{Gyr}$ an $0.8 M_{\odot}$ star brightens by $\sim 0.3$ mag in the $V$ band and $\sim 0.2 \mathrm{mag}$ in the $K$ band, moving noticeably in the Fig. 2 diagram. By $0.7 M_{\odot}$ stellar evolution effects become small, with a brightening between 8 Gyr to 12 Gyr of $\sim 0.1 \mathrm{mag}$ in both the $V$ and $K$ bands. The age/metallicity relation might therefore introduce a small systematic bias in our relation, but that would affect at most the highest mass fringe of its validity range.

The lower panels of Fig. 2 display the residuals from that relation. The absence of any obvious systematic pattern demonstrates that the calibration remains valid over its stated range. The consistency between the residuals of the WW05 measurements and ours ensures that any systematic difference between the two datasets must be small where they overlap, for approximately solar metallicities. For significantly subsolar metallicities (i.e. well below $-0.25 \mathrm{dex}$ ) we have no independent validation of the WW05 data. It should be noted however that their approach has maximal uncertainties for high metallicities, where molecular veiling is most severe. The good agreement where difficulties would be most expected suggests that the low metallicity data points are valid as well.

\section{The $\mathbf{V}$-band mass-luminosity relation}

As demonstrated by DFS00, the infrared $J$-, $H$ - and $K$-band $\mathrm{M} / \mathrm{L}$ relations are very tight and in excellent agreement with model predictions, while the $V$-band relation has a large intrinsic scatter. The contrasting dispersions were qualitatively expected from different metallicity sensitivities for the visual and infrared bands (e.g. Chabrier \& Baraffe 2000), but the extent of the effect was a surprise to most observers. Metallicity affects luminosity through a given photometric filter in two ways. First, higher metallicity decreases the bolometric luminosity for a given mass, and second, it shifts flux from the visible range to the near-IR through higher line-blanketing by $\mathrm{TiO}$ and VO molecular bands. The two mechanisms work together to decrease the luminosity of the more metal-rich stars through visible filters. In the near-IR by contrast, the redward shift of the flux distribution of the metal-rich stars counteracts their lower bolometric luminosity. The models therefore predict IR absolute magnitudes that are largely insensitive to metallicity, and the tight empirical $\mathrm{M} / \mathrm{L}$ relations confirm this. DFS00 could on the other hand not quantitatively verify their suggestion that metallicity explains the $V$-band dispersion. The Table 4 measurements now allow us to perform this verification.

Since the $K$-band $\mathrm{M} / \mathrm{L}$ relation is so tight, we can use the parallaxes and 2MASS photometry to derive accurate masses. Figure 3 displays those masses $\left(\operatorname{Mass}_{K}\right.$ ) as a function of the $M_{V}$ absolute magnitude, with symbol sizes proportional to the measured metallicity. The figure also shows the DFS00 $V$-band $\mathrm{M} / \mathrm{L}$ relation, and isometallicity contours obtained by remapping Eq. (1) to the Mass $/ M_{V}$ plane. It is immediately obvious that the position relative to the average $\mathrm{M} / \mathrm{L}$ relation correlates with metallicity, with the smallest symbols far above the $\mathrm{M} / \mathrm{L}$ relation and the largest ones under that relation.

Figure 4 provides a more quantitative view, by projecting the Mass $/ M_{V} /[\mathrm{Fe} / \mathrm{H}]$ information along the average $V$-band $\mathrm{M} / \mathrm{L}$ relation. This diagram of $[\mathrm{Fe} / \mathrm{H}]$ as a function of the difference between masses derived from the $V$ - and 

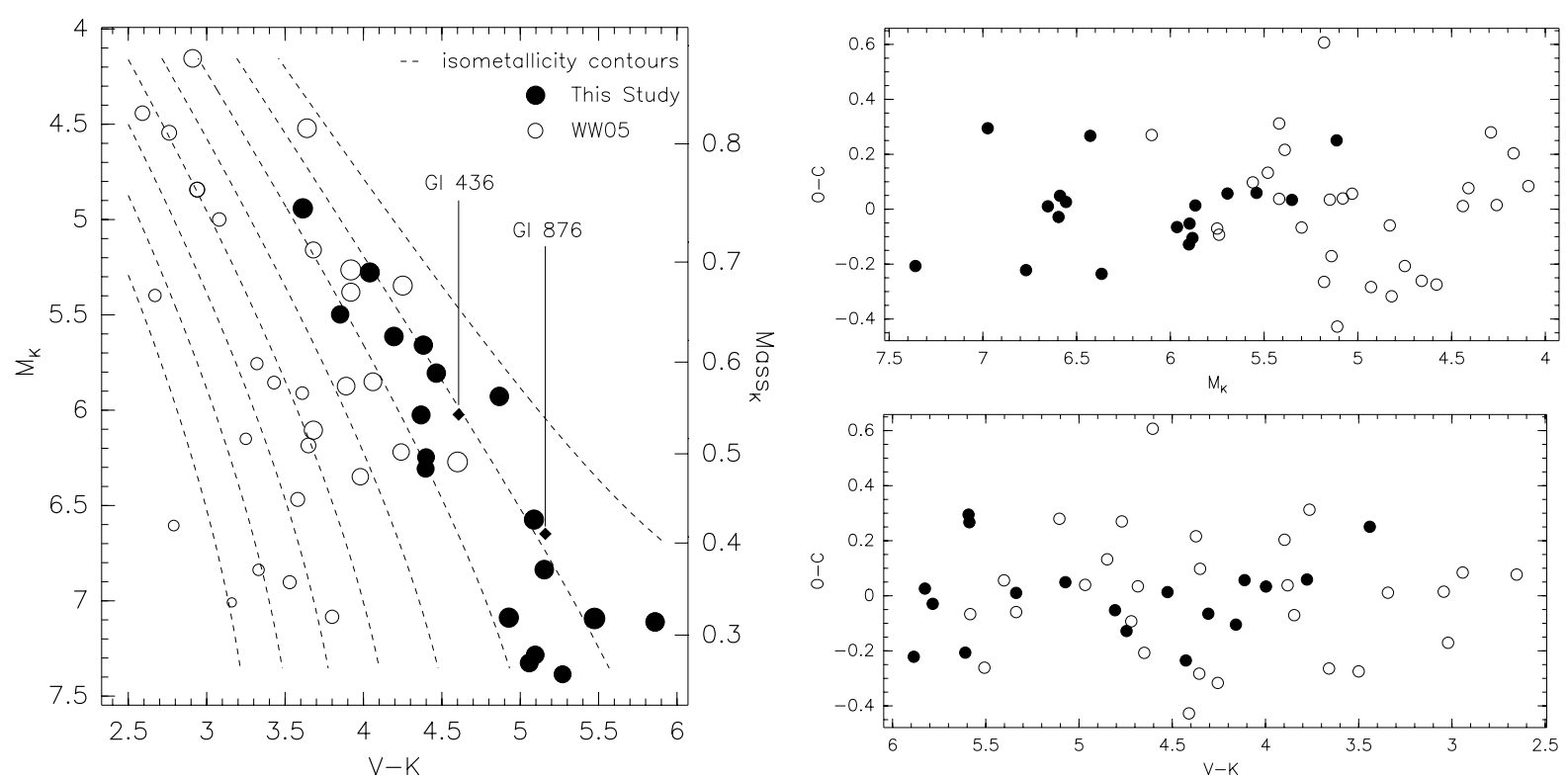

Fig. 2. Left panel: color-magnitude diagram $V-K$ vs. $M_{K}$. The filled circles correspond to our metallicity determinations and the open circles to those from WW05. The symbol size is proportional to the metallicity. The dashed lines represent isometallicity contours for the polynomial relation of Eq. (1), spaced by $0.25 \mathrm{dex}$ from $-1.50 \mathrm{dex}$ (left) to $+0.25 \mathrm{dex}$ (right). The right-hand axis shows masses from the DFS00 $K$-band Mass-luminosity, which has very low dispersion and allows to interpret the figure as a Mass-Colour-Metallicity diagram. Gl 876 and G1436, the two known M-dwarf planet-host stars, are indicated to illustrate their solar metallicity. Right panels: residuals from the calibration as a function of both $M_{K}$ and $V-K$ photometry.

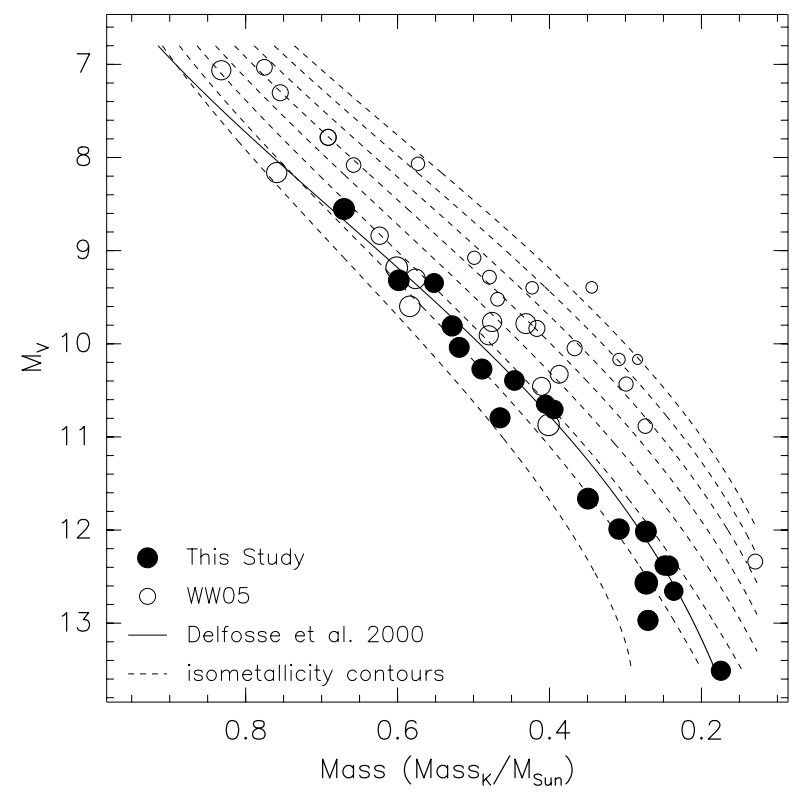

Fig. 3. $V$ band $\mathrm{M} / \mathrm{L}$ relation, with masses derived from the $K$-band $\mathrm{M} / \mathrm{L}$ relation of DFSO0 and 2MASS photometry. The filled circles represent our metallicity determinations and the open circles those from WW05. The symbol size is proportional to the metallicity, and the dashed contours represent isometallicity for the Eq. (1) calibration, spaced by 0.25 dex from +0.25 (left) to -1.5 dex (right). The solid lines represents the $V$-band empirical M/L relation of DFS00.

$K$-band $\mathrm{M} / \mathrm{L}$ relations shows a well-defined linear correlation $([\mathrm{Fe} / \mathrm{H}]=-0.149-6.508 \Delta M, \sigma([\mathrm{Fe} / \mathrm{H}])=0.21)$. This demonstrates i) that the observed dispersion indeed results primarily from a metallicity effect, and ii) that the luminosity shift

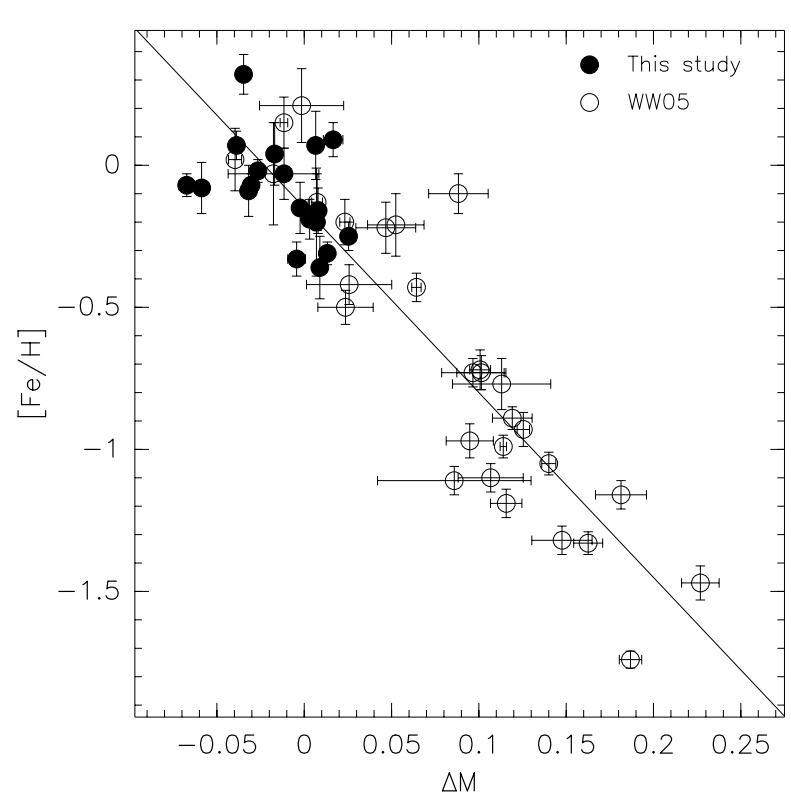

Fig. 4. Metallicity of M and K dwarfs (filled circles for our measurements, and open circles for WW05 data) as a function of the difference $(\Delta \mathrm{M})$ between masses calculated from the $V$ - and $K$-band $\mathrm{M} / \mathrm{L}$ relations of DFS00.

for a given metallicity is, to first order, constant between 0.8 and $0.2 M_{\odot}$.

We now have all the elements in hand to examine how the $V$-band luminosity depends on mass and metallicity, and to compute a mass-metallicity-luminosity relation for very-lowmass stars. We find that the $V$-band luminosity is well described 
by the following polynomial relation:

$$
\begin{aligned}
M_{V}= & 15.844-16.534 \text { Mass }+13.891 \text { Mass }^{2} \\
& -7.411 \mathrm{Mass}^{3}+1.153[\mathrm{Fe} / \mathrm{H}]
\end{aligned}
$$

for Mass $\in\left[0.2 M_{\odot}, 0.8 M_{\odot}\right]$ and $[\mathrm{Fe} / \mathrm{H}] \in[-1.5,0.2]$, and with a dispersion of 0.28 mag.

\section{Metallicity of M-dwarf planet-host stars}

It is now well established that planet host stars are more metal-rich than the average solar neighbourhood population (Gonzalez 1997; Santos et al. 2001, 2003, 2004). Santos et al. established that the planet frequency rises very steeply with stellar metallicity, at least for $[\mathrm{Fe} / \mathrm{H}]>0$. While only $\sim 3 \%$ of the solar metallicity stars are orbited by a (detected) planet, this fraction increases to over $25 \%$ for stars with $[\mathrm{Fe} / \mathrm{H}]$ above +0.3 .

One leading explanation for this dramatic dependency is that the probability of planet formation increases non-linearly with the mass of dust in a proto-planetary disk. M dwarfs, with presumably smaller disks and hence smaller disk dust mass at a given metallicity, provide a potentially critical test of that idea. This has up to now been hampered by both small statistic, with only two M-dwarf planet hosts known to date, and the lack of reliable metallicity estimates for those stars. Our calibration resolves the second of those difficulties, and shows that G1 876 and Gl 436, the two known M-dwarf planet-host stars, both have closely solar metallicities $(-0.03$ dex and +0.02 dex, respectively). Those unremarkable metal abundances do not shed light on whether M dwarf planet hosts are preferentially metalrich or not. Larger samples will be needed for that, and our calibration will be a useful tool when they become available.

\section{Metallicity distribution of $M$ dwarfs}

Equation (1) allows us to estimate the metallicity of any individual $\mathrm{M}$ dwarfs with $V$ - and $K$-band photometry and a well determined parallax. Here we use it to evaluate the metallicity distribution of the Delfosse et al. (2005, in prep.) sample of northern $\mathrm{M}$ dwarfs within 9.25 parsecs. This volume-limited sample is believed to be complete, and is therefore representative of the solar neighbourhood. We removed all unresolved binaries as well as the faintest stars which are outside the validity range of the calibration $(K \in[4 \mathrm{mag}, 7.5 \mathrm{mag}])$. Table 5 lists the $47 \mathrm{re}-$ maining stars with their estimated metallicity. For comparison, we consider a sample of 1000 non-binary solar-type stars from the CORALIE radial-velocity planet-search programme (Udry et al. 2000). This sample of single F, G or $\mathrm{K}$ dwarfs is representative of the solar neighbourhood, and we estimate their metallicity using the Santos et al. (2002) calibration of the area of the cross-correlation function between the stellar spectra and an appropriate template. We display the two distributions and their cumulative functions (Fig. 5). The two distribution have similar shapes, but with a $\sim 0.07$ dex shift of the M-dwarf distribution towards lower metallicities. A Kolmogorov-Smirnov test gives an $\sim 8 \%$ probability that the two samples are drawn from the same parent distribution. The significance of the offset is therefore modest, but if real is in the expected direction. Since

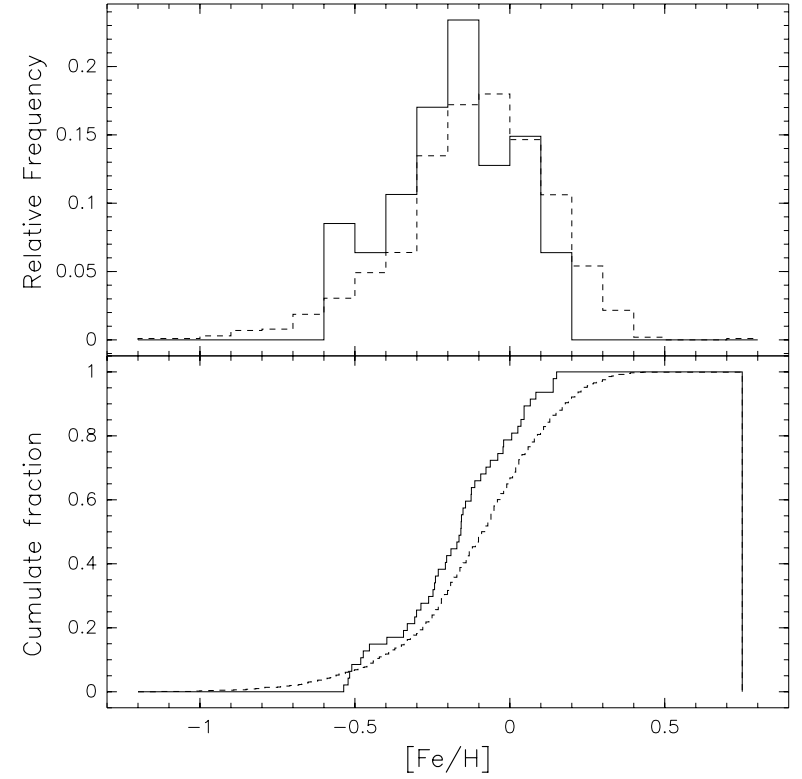

Fig. 5. Upper panel: M-dwarf metallicity distribution derived from Eq. (1) and, over-plotted in dashed line, the metallicity distribution of 1000 non-variable stars of our CORALIE radial-velocity planetsearch programme. Bottom panel: cumulative distributions of the same samples.

M-dwarfs have much longer lifetimes than the age of the universe, every M-dwarf that ever formed is still here for us to see, while some of the oldest solar-type stars have evolved to white dwarfs. M dwarfs are thus expected to be slightly older on average, and from the age-metallicity relation therefore slightly more metal-poor.

\section{Conclusions}

We have determined the metallicities of $20 \mathrm{M}$ dwarfs in widebinary systems that also contain an F, G or K star, under the simple assumption that the two stars have the same composition. Where the parameter spaces overlap, our results are consistent with the direct analysis of M-dwarf spectra by Woolf \& Wallerstein (2005). This provides a welcome validation of both our assumptions of a common composition and of the WW05 analysis of complex M-dwarf spectra. The two datasets cover complementary parameter ranges, and we join them to derive a photometric calibration of very low-mass star metallicities. The calibration is valid between 0.8 and $0.2 M_{\odot}$, needs $V$ and $K$-band photometry and an accurate parallax, and provides metallicity estimates with $\sim 0.2$ dex uncertainties. A $5 \%$ parallax uncertainty results in an additional $\sim 0.2$ dex metallicity uncertainty, making the relation useful only within $\sim 50 \mathrm{pc}$.

We use the new metallicities to take a fresh look at the $V$-band mass-luminosity relation, and demonstrate that its intrinsic dispersion is indeed due to metallicity. We apply the new calibration to the two known M dwarfs that host planets, Gl 876 and $\mathrm{Gl} 436$, and find both of solar metallicity. Larger samples of M-dwarf planet hosts will be needed to investigate whether they are preferentially metal-rich, as are their solar-type counterparts. Finally, we estimate metallicities for a volume-limited sample of $47 \mathrm{M}$ dwarfs, and compare its metallicity distribution 
to that of a much larger sample of solar-type stars. The difference between the two distributions is small, but if real might reflect slightly older average ages for the long-lived M-dwarfs. In a forthcoming paper we will publish metallicities for a larger sample of M-dwarfs in binaries, observed from the southern hemisphere, and will attempt to derive a purely spectrophotometric metallicity calibration.

Acknowledgements. We would like to acknoledge the anonymous referee for constructive comments which led to an improved paper. This research has made use of the SIMBAD database, operated at CDS, Strasbourg, France. This publication makes use of data products from the Two Micron All Sky Survey, which is a joint project of the University of Massachusetts and the Infrared Processing and Analysis Center/California Institute of Technology, funded by the National Aeronautics and Space Administration and the National Science Foundation.

\section{References}

Allard, F., \& Hauschildt, P. H. 1995, ApJ, 445, 433

Alonso, A., Arribas, S., \& Martinez-Roger, C. 1996, A\&A, 313, 873

Baranne, A., Queloz, D., Mayor, M., et al. 1996, A\&AS, 119, 373

Bonfils, X., Delfosse, X., Udry, S., et al. 2004, PASP, 318, 286

Butler, R. P., Vogt, S., Marcy, G. W., et al. 2004

[arXiv: astro-ph/8587]

Carpenter, J. M. 2001, AJ, 121, 2851

Chabrier, G., \& Baraffe, I. 2000, ARA\&A, 38, 337

Chabrier, G. 2003, PASP, 115, 763

Dawson, P. C., \& De Robertis, M. M. 2004, AJ, 127, 2909

Delfosse, X., Forveille, T., Mayor, et al. 1998, A\&A, 338, L67

Delfosse, X., Forveille, T., Ségransan, D., et al. 2000, A\&A, 364, 217

Edvardsson, B., Andersen, J., \& Gustafsson, B. 1993, A\&A, 275, 101

Egret, D., Didelon, P., McLean, B. J., et al. 1992, A\&A, 258, 217

Endl, M., Cochran, W. D., Tull, R. G., et al. 2003, AJ, 126, 3099

ESA 1997, The Hipparcos and Tycho Catalogues, ESA SP-1200, Noordwijk: ESA)

Fleming, T. A., Schmitt, J. H. M. M., \& Giampapa, M. S. 1995, ApJ, 450,401

Gizis, J. E. 1997, AJ, 113, 806

Gizis, J. E., \& Reid, N. 1997, PASP, 109, 1233

Gliese, W., \& Jahreiss, H. 1991, STIA, 224, 161
Gonzalez, G. 1997, MNRAS, 285, 403

Gonzalez, G., \& Vanture, A. D. 1998, A\&A, 339, L29

Gould, A., \& Chanamé, J. 2004, ApJS, 150, 455

Ida, S., \& Lin, D. N. C. 2005 [arXiv:astro-ph/2566]

Jones, H. R. A., Longmore, A. J., Allard, F., et al. 1996, MNRAS, 280, 77

Jones, H. R. A., Pavlenko, Y., Viti, S., et al. 2002, MNRAS, 330, 675

Kuerster, M., Endl, M., Rouesnel, F., et al. 2003, A\&A, 403, 1077

Kurucz, R. L. 1993, CD-ROMs, ATLAS9 Stellar Atmospheres Programs and $2 \mathrm{~km} \mathrm{~s}^{-1}$ Grid (Cambridge: Smithsonian Astrophys. Obs.)

Laughlin, G., Bodenheimer, P., \& Adams, F. C. 2004, ApJ, 612, L73

Leggett, S. 1992, ApJS, 82, 351

Marcy, G. W., Butler, R. P., Vogt, S. S., et al. 1998, ApJ, 505, L147

Marcy, G. W., Butler, R. P., Fischer, D., et al. 2001, ApJ, 556, 296

Mermilliod, J.-C., Mermilliod, M., \& Hauck, B. 1997, A\&AS, 124, 349

Mould, J. R. 1976, AJ, 210, 402

Mould, J. R. 1978, ApJ, 226, 923

Olsen, E. H. 1994, A\&AS, 106, 257

Poveda, A., Herrera, M. A., Allen, C., et al. 1994, Rev. Mex. Astron. Astrofis., 28, 43

Press, W. H., Teukolsky, S. A., Vetterling, W. T., et al. 1992, in Numerical Recipes in Fortran 77, Volume 1 (Cambridge University Press), Second Edition

Santos, N. C., Israelian, G., \& Mayor, M. 2001, A\&A, 373, 1019

Santos, N. C., Mayor, M., Naef, D., et al. 2002, A\&A, 392, 215

Santos, N. C., Israelian, G., Mayor, M., et al. 2003, A\&A, 398, 363

Santos, N. C., Israelian, G., \& Mayor, M. 2004, A\&A, 415, 1153

Santos, N. C., Israelian, G., Mayor, M., et al. 2005, A\&A, in press

Ségransan, D., Kervella, P., Forveille, T., et al. 2003, A\&A, 397, L5

Sneden, C. 1973, Ph.D. Thesis, University of Texas

Udry, S., Mayor, M., Naef, D., et al. 2000, A\&A, 356, 590

Valenti, J. A., Piskunov, N., \& Johns-Krull, C. M. 1998, ApJ, 498, 851

van Altena, W. F., Lee, J. T., \& Hoffleit, E. D. 1995, The General Catalogue of Trigonometric Stellar Parallaxes, 4th ed. (Schenectady, NY: L. Davis Press)

Weis, E. W. 1991, AJ, 102, 1795

Woolf, V. M., \& Wallerstein, G. 2004, MNRAS, 350, 575

Woolf, V. M., \& Wallerstein, G. 2004, MNRAS, 356, 963

Wright, J. T., Marcy, G. W., Butler, R. P., et al. 2004, ApJS, 152, 261

Zboril, M., \& Byrne, P. B. 1998, MNRAS, 299, 753 


\section{Online Material}


X. Bonfils et al.: Metallicity of M dwarfs. I., Online Material p 2

Table 4. Apparent magnitudes, parallaxes, masses derived from the M/L relations of DFS00, and metallicities from this study and from WW05.

\begin{tabular}{|c|c|c|c|c|c|c|c|c|c|c|}
\hline Star & $\begin{array}{l}\text { Spectral } \\
\text { type }\end{array}$ & $\begin{array}{c}V \\
{[\mathrm{mag}]}\end{array}$ & $\begin{array}{c}\text { source } \\
V^{\dagger}\end{array}$ & $\begin{array}{c}K \\
{[\mathrm{mag}]}\end{array}$ & $\begin{array}{c}\pi \\
{[\mathrm{mas}]}\end{array}$ & $\begin{array}{c}\text { source } \\
\pi^{\dagger \dagger}\end{array}$ & $\begin{array}{c}\operatorname{Mass}_{V} \\
{\left[M_{\odot}\right]}\end{array}$ & $\begin{array}{c}\operatorname{Mass}_{K} \\
{\left[M_{\odot}\right]}\end{array}$ & $\begin{array}{c}{[\mathrm{Fe} / \mathrm{H}]} \\
{[\mathrm{dex}]}\end{array}$ & $\begin{array}{c}\text { source } \\
{[\mathrm{Fe} / \mathrm{H}]^{\ddagger}}\end{array}$ \\
\hline CD-48 176B & $\mathrm{M}$ & 13.50 & $\mathrm{~S}$ & $7.64 \pm 0.02$ & $66.92 \pm 0.73$ & $\mathrm{H}$ & 0.212 & 0.270 & $-0.08 \pm 0.09$ & $\mathrm{a}$ \\
\hline GJ 3195 B & M3 & 12.50 & $\mathrm{G}$ & $8.10 \pm 0.03$ & $43.74 \pm 0.84$ & $\mathrm{H}$ & 0.408 & 0.395 & $-0.31 \pm 0.04$ & $\mathrm{a}$ \\
\hline GJ 3825 & esdM1.5 & $14.55 \pm 0.03$ & M & $10.86 \pm 0.01$ & $36.1 \pm 3.2$ & $\mathrm{Y}$ & 0.255 & 0.129 & $-0.93 \pm 0.06$ & $\mathrm{~b}$ \\
\hline GJ 687 & M3 & $9.15 \pm 0.03$ & M & $4.55 \pm 0.02$ & $220.9 \pm 0.9$ & $\mathrm{H}$ & 0.389 & 0.401 & $+0.15 \pm 0.09$ & $\mathrm{~b}$ \\
\hline GJ 9192 & K4 & $10.70 \pm 0.02$ & M & $7.76 \pm 0.02$ & $26.1 \pm 2.1$ & $\mathrm{H}$ & 0.793 & 0.691 & $-0.73 \pm 0.06$ & $\mathrm{~b}$ \\
\hline GJ 9371 & sdM0.0 & $12.20 \pm 0.03$ & M & $8.67 \pm 0.02$ & $44.3 \pm 2.8$ & $\mathrm{H}$ & 0.439 & 0.299 & $-1.05 \pm 0.04$ & $\mathrm{~b}$ \\
\hline G 22-15 & K5V & $9.23 \pm 0.02$ & M & $6.47 \pm 0.02$ & $41.2 \pm 1.3$ & $\mathrm{H}$ & 0.855 & 0.754 & $-0.72 \pm 0.07$ & $\mathrm{~b}$ \\
\hline Gl 105 B & M4 & $11.67 \pm 0.01$ & M & $6.57 \pm 0.02$ & $138.72 \pm 1.04$ & $\mathrm{H}$ & 0.252 & 0.248 & $-0.19 \pm 0.07$ & $\mathrm{a}$ \\
\hline Gl 107 B & M1.5 & 10.06 & $\mathrm{G}$ & $5.87 \pm 0.02$ & $89.03 \pm 0.79$ & $\mathrm{H}$ & 0.517 & 0.528 & $-0.03 \pm 0.09$ & $\mathrm{a}$ \\
\hline Gl $166 \mathrm{C}$ & M4.5 & 11.17 & $\mathrm{G}$ & $5.9 \pm 0.10$ & $198.25 \pm 0.84$ & $\mathrm{H}$ & 0.232 & 0.236 & $-0.33 \pm 0.06$ & $\mathrm{a}$ \\
\hline Gl 191 & sdM1.0 & $8.85 \pm 0.03$ & M & $5.05 \pm 0.02$ & $255.1 \pm 0.9$ & $\mathrm{H}$ & 0.388 & 0.274 & $-0.99 \pm 0.04$ & $\mathrm{~b}$ \\
\hline Gl 205 & M1.5 & $7.96 \pm 0.01$ & M & $4.04 \pm 0.26$ & $175.7 \pm 1.2$ & $\mathrm{H}$ & 0.600 & 0.601 & $+0.21 \pm 0.13$ & $\mathrm{~b}$ \\
\hline G1 212 & M0.5 & $9.80 \pm 0.01$ & $\mathrm{~T}$ & $5.76 \pm 0.02$ & $80.13 \pm 1.67$ & $\mathrm{H}$ & 0.572 & 0.598 & $+0.04 \pm 0.11$ & $\mathrm{a}$ \\
\hline Gl 231.1 B & M3.5 & 13.42 & $\mathrm{G}$ & $8.28 \pm 0.02$ & $51.76 \pm 0.78$ & $\mathrm{H}$ & 0.282 & 0.309 & $-0.02 \pm 0.04$ & $\mathrm{a}$ \\
\hline G1 250 B & M2 & 10.09 & $\mathrm{G}$ & $5.72 \pm 0.04$ & $114.94 \pm 0.86$ & $\mathrm{H}$ & 0.442 & 0.446 & $-0.15 \pm 0.09$ & $\mathrm{a}$ \\
\hline Gl 297.2 B & M2 & $11.80 \pm 0.01$ & M & $7.42 \pm 0.02$ & $44.47 \pm 0.77$ & $\mathrm{H}$ & 0.484 & 0.519 & $-0.09 \pm 0.09$ & $\mathrm{a}$ \\
\hline Gl 324 B & M3.5 & 13.14 & G & $7.67 \pm 0.02$ & $76.8 \pm 0.84$ & $\mathrm{H}$ & 0.239 & 0.273 & $+0.32 \pm 0.07$ & $\mathrm{a}$ \\
\hline Gl 380 & K5 & $6.60 \pm 0.02$ & M & $2.96 \pm 0.29$ & $205.2 \pm 0.8$ & $\mathrm{H}$ & 0.742 & 0.759 & $-0.03 \pm 0.18$ & $\mathrm{~b}$ \\
\hline Gl 411 & M2V & $7.49 \pm 0.02$ & M & $3.25 \pm 0.31$ & $392.5 \pm 0.9$ & $\mathrm{H}$ & 0.436 & 0.410 & $-0.42 \pm 0.07$ & $\mathrm{~b}$ \\
\hline Gl 412 A & M0.5 & $8.75 \pm 0.04$ & M & $4.77 \pm 0.02$ & $206.9 \pm 1.2$ & $\mathrm{H}$ & 0.451 & 0.387 & $-0.43 \pm 0.05$ & $\mathrm{~b}$ \\
\hline Gl 414 B & M1.5 & $9.98 \pm 0.04$ & M & $5.73 \pm 0.02$ & $83.8 \pm 1.1$ & $\mathrm{H}$ & 0.544 & 0.584 & $+0.02 \pm 0.11$ & $\mathrm{~b}$ \\
\hline G1 505 B & M0.5 & 9.60 & $\mathrm{G}$ & $5.75 \pm 0.02$ & $89.07 \pm 0.99$ & $\mathrm{H}$ & 0.554 & 0.552 & $-0.25 \pm 0.05$ & $\mathrm{a}$ \\
\hline G1 506.1 & $\mathrm{sdK}$ & $10.84 \pm 0.02$ & M & $8.17 \pm 0.02$ & $27.9 \pm 2.5$ & $\mathrm{H}$ & 0.754 & 0.573 & $-1.16 \pm 0.05$ & $\mathrm{~b}$ \\
\hline G1 526 & M1.5 & $8.46 \pm 0.01$ & M & $4.42 \pm 0.21$ & $184.1 \pm 1.3$ & $\mathrm{H}$ & 0.520 & 0.431 & $-0.10 \pm 0.07$ & $\mathrm{~b}$ \\
\hline Gl 53.1 B & M3 & 13.60 & $\mathrm{G}$ & $8.67 \pm 0.02$ & $48.2 \pm 1.06$ & $\mathrm{H}$ & 0.280 & 0.273 & $+0.07 \pm 0.12$ & $\mathrm{a}$ \\
\hline Gl 544 B & M6 & 15.10 & $\mathrm{G}$ & $9.59 \pm 0.02$ & $48.12 \pm 1.11$ & $\mathrm{H}$ & 0.215 & 0.174 & $-0.20 \pm 0.19$ & $\mathrm{a}$ \\
\hline G1 701 & M1 & $9.37 \pm 0.03$ & M & $5.31 \pm 0.02$ & $128.3 \pm 1.4$ & $\mathrm{H}$ & 0.503 & 0.480 & $-0.20 \pm 0.08$ & $\mathrm{~b}$ \\
\hline G1 768.1 B & M3.5 & 13.10 & $\mathrm{G}$ & $8.01 \pm 0.03$ & $51.57 \pm 0.77$ & $\mathrm{H}$ & 0.310 & 0.349 & $+0.07 \pm 0.12$ & $\mathrm{a}$ \\
\hline G1 783.2 B & M4 & 13.94 & $\mathrm{G}$ & $8.88 \pm 0.02$ & $48.83 \pm 0.91$ & $\mathrm{H}$ & 0.251 & 0.243 & $-0.16 \pm 0.08$ & $\mathrm{a}$ \\
\hline G1 797 B & M2.5 & 11.88 & $\mathrm{G}$ & $7.42 \pm 0.02$ & $47.65 \pm 0.76$ & $\mathrm{H}$ & 0.460 & 0.489 & $-0.07 \pm 0.04$ & a \\
\hline G1 809 & M0.5 & $8.54 \pm 0.04$ & M & $4.62 \pm 0.02$ & $142.0 \pm 0.8$ & $\mathrm{H}$ & 0.584 & 0.576 & $-0.13 \pm 0.10$ & $\mathrm{~b}$ \\
\hline G1 81.1 B & $\mathrm{K} 7$ & 11.21 & $\mathrm{G}$ & $7.60 \pm 0.03$ & $29.43 \pm 0.91$ & $\mathrm{H}$ & 0.684 & 0.671 & $+0.09 \pm 0.06$ & $\mathrm{a}$ \\
\hline G1 872 B & M1 & 11.70 & G & $7.30 \pm 0.02$ & $61.54 \pm 0.77$ & $\mathrm{H}$ & 0.414 & 0.405 & $-0.36 \pm 0.11$ & a \\
\hline G1 887 & M0.5 & $7.35 \pm 0.02$ & M & $3.46 \pm 0.20$ & $303.9 \pm 0.9$ & $\mathrm{H}$ & 0.522 & 0.476 & $-0.22 \pm 0.09$ & $\mathrm{~b}$ \\
\hline LHS 104 & esdK7 & $13.74 \pm 0.02$ & M & $10.4 \pm 0.02$ & $19.3 \pm 3.0$ & $\mathrm{Y}$ & 0.471 & 0.308 & $-1.33 \pm 0.04$ & $\mathrm{~b}$ \\
\hline LHS 12 & M0.5 & $12.26 \pm 0.04$ & M & $8.68 \pm 0.02$ & $36.1 \pm 4.3$ & $\mathrm{H}$ & 0.486 & 0.367 & $-0.89 \pm 0.04$ & $\mathrm{~b}$ \\
\hline LHS 170 & $\mathrm{sdK}$ & $10.68 \pm 0.01$ & M & $7.60 \pm 0.02$ & $30.2 \pm 2.4$ & $\mathrm{H}$ & 0.753 & 0.658 & $-0.97 \pm 0.06$ & $\mathrm{~b}$ \\
\hline LHS 173 & sdK7 & $11.11 \pm 0.01$ & M & $7.79 \pm 0.02$ & $39.2 \pm 2.5$ & $\mathrm{H}$ & 0.615 & 0.499 & $-1.19 \pm 0.05$ & $\mathrm{~b}$ \\
\hline LHS 174 & sdM0.5 & $12.75 \pm 0.01$ & M & $9.14 \pm 0.02$ & $22.6 \pm 7.4$ & $\mathrm{Y}$ & 0.554 & 0.468 & $-1.11 \pm 0.05$ & $\mathrm{~b}$ \\
\hline LHS 1819 & K4 & $10.88 \pm 0.02$ & M & $8.29 \pm 0.03$ & $17.0 \pm 2.6$ & $\mathrm{H}$ & 0.888 & 0.775 & $-0.77 \pm 0.09$ & $\mathrm{~b}$ \\
\hline LHS 1841 & K & $13.18 \pm 0.03$ & M & $10.39 \pm 0.02$ & $17.5 \pm 3.3$ & $\mathrm{Y}$ & 0.571 & 0.344 & $-1.47 \pm 0.06$ & $\mathrm{~b}$ \\
\hline LHS 236 & $\operatorname{sdK} 7$ & $13.10 \pm 0.01$ & M & $9.85 \pm 0.02$ & $18.2 \pm 2.9$ & $\mathrm{Y}$ & 0.570 & 0.423 & $-1.32 \pm 0.05$ & $\mathrm{~b}$ \\
\hline LHS 2938 & K7 & $10.67 \pm 0.02$ & M & $7.76 \pm 0.02$ & $19.0 \pm 2.0$ & $\mathrm{H}$ & 0.885 & 0.832 & $-0.21 \pm 0.11$ & $\mathrm{~b}$ \\
\hline LHS 3084 & $\mathrm{sdK}$ & $13.43 \pm 0.03$ & M & $9.78 \pm 0.02$ & $19.1 \pm 2.9$ & $\mathrm{Y}$ & 0.513 & 0.416 & $-0.73 \pm 0.05$ & $\mathrm{~b}$ \\
\hline LHS 343 & $\mathrm{sdK}$ & $13.82 \pm 0.02$ & M & $10.66 \pm 0.02$ & $18.6 \pm 3.7$ & $\mathrm{Y}$ & 0.471 & 0.284 & $-1.74 \pm 0.03$ & $\mathrm{~b}$ \\
\hline LHS 467 & esdK7 & $12.21 \pm 0.03$ & M & $8.78 \pm 0.02$ & $26.0 \pm 3.6$ & $\mathrm{H}$ & 0.586 & 0.479 & $-1.10 \pm 0.05$ & $\mathrm{~b}$ \\
\hline LHS 5337 & M0 & $11.15 \pm 0.03$ & M & $7.47 \pm 0.02$ & $34.5 \pm 3.3$ & $\mathrm{H}$ & 0.647 & 0.624 & $-0.50 \pm 0.06$ & $\mathrm{~b}$ \\
\hline NLTT 45791 & M & 13.10 & $\mathrm{~N}$ & $8.23 \pm 0.03$ & $34.57 \pm 0.73$ & $\mathrm{G}$ & 0.398 & 0.465 & $-0.07 \pm 0.05$ & $\mathrm{a}$ \\
\hline
\end{tabular}

† M: Mermilliod et al. (1997), G: Gliese \& Jareiss (1991), N: Gould \& Chanamé (2004), T: Tycho Input Catalogue (Egret et al. 1992), S: Simbad database. Unfortunately their is no uncertainties on $V$-band photometry in the Gliese \& Jareiss catalogue.

${ }^{\dagger \dagger}$ H: ESA (1997), Y: van Altena et al. (1995), G: Gould \& Chanamé (2004).

$\ddagger$ a: this paper, b: Woolf \& Wallerstein (2005).

The $K$-band photometry is from 2MASS. The mass estimates use the listed photometry and the M/L relations of Delfosse et al. (2000). 
X. Bonfils et al.: Metallicity of M dwarfs. I., Online Material p 3

Table 5. Magnitudes, parallaxes, corresponding masse and metallicity estimates of M-dwarf neighbors.

\begin{tabular}{|c|c|c|c|c|c|c|c|c|}
\hline Star & $\begin{array}{l}\text { Spectral } \\
\text { type }\end{array}$ & $\begin{array}{c}V \\
{[\mathrm{mag}]}\end{array}$ & $\begin{array}{c}\text { source } \\
V^{\dagger}\end{array}$ & $\begin{array}{c}K \\
{[\mathrm{mag}]}\end{array}$ & $\begin{array}{c}\pi \\
{[\mathrm{mas}]}\end{array}$ & $\begin{array}{l}M_{\star, V} \\
{\left[M_{\odot}\right]}\end{array}$ & $\begin{array}{l}M_{\star, K} \\
{\left[M_{\odot}\right]}\end{array}$ & $\begin{array}{l}\mathrm{Fe} / \mathrm{H}] \\
{[\mathrm{dex}]}\end{array}$ \\
\hline G115A & M1 & $8.10 \pm 0.02$ & $\mathrm{M}$ & $4.02 \pm 0.02$ & $280.30 \pm 1.00$ & 0.450 & 0.404 & -0.45 \\
\hline G148 & M3 & $10.05 \pm 0.01$ & M & $5.45 \pm 0.02$ & $122.80 \pm 1.20$ & 0.438 & 0.471 & +0.04 \\
\hline Gl109 & M3 & $10.60 \pm 0.01$ & M & $5.96 \pm 0.02$ & $132.40 \pm 2.50$ & 0.354 & 0.350 & -0.20 \\
\hline G1205 & M1.5 & $7.97 \pm 0.01$ & M & $4.04 \pm 0.26$ & $175.70 \pm 1.20$ & 0.594 & 0.602 & -0.09 \\
\hline LHS1805 & M3.5 & 11.71 & $\mathrm{G}$ & $6.64 \pm 0.02$ & $132.10 \pm 4.90$ & 0.257 & 0.253 & -0.16 \\
\hline Gl251 & M3V & $10.02 \pm 0.01$ & M & $5.28 \pm 0.02$ & $181.30 \pm 1.90$ & 0.346 & 0.350 & -0.16 \\
\hline G1273 & M3.5 & $9.84 \pm 0.01$ & M & $4.86 \pm 0.02$ & $263.30 \pm 1.40$ & 0.289 & 0.291 & -0.16 \\
\hline G1285 & M4.5 & $11.21 \pm 0.02$ & M & $5.70 \pm 0.02$ & $168.60 \pm 2.70$ & 0.262 & 0.309 & +0.07 \\
\hline GJ1105 & M3.5 & $12.01 \pm 0.01$ & M & $6.88 \pm 0.03$ & $120.80 \pm 4.40$ & 0.249 & 0.248 & -0.15 \\
\hline GJ2066 & M2 & $10.12 \pm 0.01$ & M & $5.77 \pm 0.02$ & $109.20 \pm 1.80$ & 0.456 & 0.459 & -0.14 \\
\hline Gl382 & M1.5 & $9.26 \pm 0.01$ & M & $5.01 \pm 0.02$ & $128.00 \pm 1.50$ & 0.526 & 0.541 & -0.02 \\
\hline Gl388 & M3 & $9.41 \pm 0.02$ & M & $4.59 \pm 0.02$ & $204.60 \pm 2.80$ & 0.391 & 0.423 & +0.05 \\
\hline G1393 & M2 & $9.66 \pm 0.01$ & M & $5.31 \pm 0.02$ & $138.30 \pm 2.10$ & 0.454 & 0.448 & -0.17 \\
\hline Gl402 & M4 & $11.66 \pm 0.01$ & M & $6.37 \pm 0.02$ & $145.90 \pm 3.80$ & 0.249 & 0.260 & -0.06 \\
\hline G1408 & M2.5 & $10.04 \pm 0.01$ & M & $5.34^{\ddagger}$ & $151.00 \pm 1.60$ & 0.387 & 0.372 & -0.24 \\
\hline Gl411 & M2 & $7.49 \pm 0.04$ & M & $3.25 \pm 0.31$ & $392.40 \pm 0.90$ & 0.435 & 0.410 & -0.33 \\
\hline G1412A & M0.5 & $8.82 \pm 0.01$ & M & $4.77 \pm 0.02$ & $206.90 \pm 1.20$ & 0.458 & 0.387 & -0.51 \\
\hline Gl424 & M0 & $9.30 \pm 0.01$ & M & $5.53 \pm 0.02$ & $109.90 \pm 1.10$ & 0.556 & 0.502 & -0.47 \\
\hline Gl445 & M3.5 & $10.84 \pm 0.01$ & M & $5.95 \pm 0.03$ & $185.50 \pm 1.40$ & 0.270 & 0.247 & -0.26 \\
\hline Gl450 & M1 & $9.74 \pm 0.03$ & M & $5.61 \pm 0.02$ & $116.90 \pm 1.40$ & 0.487 & 0.462 & -0.29 \\
\hline Gl486 & M3.5 & $11.38 \pm 0.01$ & M & $6.36 \pm 0.02$ & $121.80 \pm 2.90$ & 0.305 & 0.316 & -0.08 \\
\hline G1514 & M0.5 & $9.04 \pm 0.01$ & M & $5.04 \pm 0.03$ & $131.10 \pm 1.30$ & 0.545 & 0.526 & -0.23 \\
\hline LHS2784 & M3.5 & $11.97 \pm 0.02$ & $\mathrm{~W}$ & $6.98 \pm 0.02$ & $109.90 \pm 3.20$ & 0.265 & 0.261 & -0.19 \\
\hline G1526 & M1.5 & $8.48 \pm 0.03$ & M & $4.42 \pm 0.02$ & $184.10 \pm 1.30$ & 0.527 & 0.502 & -0.24 \\
\hline G1555 & M4 & $11.30 \pm 0.02$ & M & $5.94 \pm 0.03$ & $163.50 \pm 2.80$ & 0.252 & 0.284 & +0.01 \\
\hline Gl581 & M3 & $10.55 \pm 0.01$ & M & $5.84 \pm 0.02$ & $159.50 \pm 2.30$ & 0.324 & 0.307 & -0.25 \\
\hline Gl625 & M1.5 & $10.11 \pm 0.01$ & M & $5.83 \pm 0.02$ & $151.90 \pm 1.10$ & 0.375 & 0.323 & -0.48 \\
\hline Gl628 & M3.5 & $10.07 \pm 0.02$ & M & $5.08 \pm 0.02$ & $234.50 \pm 1.80$ & 0.294 & 0.296 & -0.12 \\
\hline Gl687 & M3 & $9.16 \pm 0.02$ & M & $4.55 \pm 0.02$ & $220.80 \pm 0.90$ & 0.343 & 0.401 & +0.11 \\
\hline Gl686 & M1 & $9.60 \pm 0.02$ & M & $5.57 \pm 0.02$ & $123.00 \pm 1.60$ & 0.486 & 0.447 & -0.40 \\
\hline G1701 & M1 & $9.36 \pm 0.01$ & M & $5.31 \pm 0.02$ & $128.30 \pm 1.40$ & 0.499 & 0.481 & -0.30 \\
\hline GJ1230B & M5 & 14.00 & $\mathrm{G}$ & $6.62 \pm 0.02$ & $120.90 \pm 7.20$ & 0.143 & 0.281 & +0.15 \\
\hline G1725A & M3 & $8.95 \pm 0.01$ & M & $4.43 \pm 0.02$ & $280.30 \pm 3.60$ & 0.361 & 0.334 & -0.31 \\
\hline G1725B & M3.5 & $9.72 \pm 0.01$ & M & $5.00 \pm 0.02$ & $284.50 \pm 5.00$ & 0.292 & 0.250 & -0.34 \\
\hline G1745A & M1.5 & 10.76 & $\mathrm{G}$ & $6.52 \pm 0.02$ & $115.90 \pm 2.50$ & 0.373 & 0.308 & -0.54 \\
\hline G1745B & M2 & 10.75 & $\mathrm{G}$ & $6.52 \pm 0.02$ & $112.80 \pm 2.40$ & 0.380 & 0.317 & -0.52 \\
\hline G1752A & $\mathrm{M} 2.5$ & $9.13 \pm 0.01$ & M & $4.67 \pm 0.02$ & $170.30 \pm 1.40$ & 0.460 & 0.484 & -0.02 \\
\hline G1793 & M2.5 & $10.63 \pm 0.01$ & M & $5.93 \pm 0.02$ & $125.60 \pm 1.10$ & 0.376 & 0.374 & -0.12 \\
\hline Gl809 & M0.5 & $8.55 \pm 0.02$ & M & $4.62 \pm 0.02$ & $141.90 \pm 0.80$ & 0.589 & 0.577 & -0.16 \\
\hline G1849 & M3.5 & $10.37 \pm 0.01$ & M & $5.59 \pm 0.02$ & $114.00 \pm 2.10$ & 0.410 & 0.475 & +0.14 \\
\hline G1860A & M3 & 9.85 & $\mathrm{G}$ & $4.78 \pm 0.03$ & $247.50 \pm 1.50$ & 0.292 & 0.322 & -0.04 \\
\hline G1873 & M3.5 & $10.05 \pm 0.01$ & M & $5.30 \pm 0.02$ & $198.10 \pm 2.00$ & 0.300 & 0.317 & -0.21 \\
\hline G1876 & M4 & $10.18 \pm 0.01$ & M & $5.01 \pm 0.02$ & $214.60 \pm 0.20$ & 0.293 & 0.334 & +0.03 \\
\hline G1880 & M1.5 & $8.70 \pm 0.02$ & M & $4.52 \pm 0.02$ & $145.30 \pm 1.20$ & 0.569 & 0.586 & +0.05 \\
\hline G1896A & M3.5 & 10.38 & $\mathrm{G}$ & $5.33 \pm 0.02$ & $160.10 \pm 2.80$ & 0.333 & 0.387 & +0.08 \\
\hline G1896B & M4.5 & 12.40 & $\mathrm{G}$ & $6.26 \pm 0.04$ & $160.10 \pm 2.80$ & 0.186 & 0.248 & +0.14 \\
\hline G1908 & M1 & $8.99 \pm 0.01$ & M & $5.04 \pm 0.02$ & $167.50 \pm 1.50$ & 0.479 & 0.421 & -0.52 \\
\hline
\end{tabular}

† M: Mermilliod et al. (1997), G: Gliese \& Jareiss (1991), W: Weis (1991).

${ }^{*} K$-band photometry given by Leggett (1992) and transformed to 2MASS system using the relation given by Carpenter (2001).

The mass estimates use the listed photometry and the M/L relations of Delfosse et al. (2000).

The $K$-band photometry originates from 2MASS and the parallaxes are adopted from the compilation of Delfosse et al. (2005, in prep.). 